\title{
New hope in the fight against malaria?
}

\author{
"...the severe malaria situation has encouraged the scientific community to try to find new drugs to tackle this \\ disease. In fact, several nonprofit, public-private partnerships such as Medicines for Malaria Venture and some \\ pharmaceutical companies are currently in the process of lead generation or optimization."
}

Keywords: high-throughput screening $=$ liver stage $\approx$ malaria $\approx$ mitochondrial electron transport chain

Malaria is still one of the major vector-borne infectious diseases in the world. The most recent report from the WHO states that malaria is responsible for approximately one million deaths per year, mainly of children below the age of five [101]. Since this disease is endemic in developing countries, it had traditionally drawn little attention from public and private resources in comparison to other maladies. Nowadays, the rapid emergence of multi-resistant Plasmodium falciparum strains, the most virulent species of malaria parasites, is impairing both prophylaxis and chemotherapy. In addition, we are running late as regards to therapeutic solutions, as the first signs of resistance to artemisinin have been recently reported [1]. This is particularly worrying given that no other drug options or vaccines are available and 15 years have passed since we last saw the introduction of a new drug class onto the clinical stage [2]. Consequently, the severe malaria situation has encouraged the scientific community to try to find new drugs to tackle this disease. In fact, several nonprofit, public-private partnerships such as Medicines for Malaria Venture (MMV) [102] and some pharmaceutical companies are currently in the process of lead generation or optimization.

In recent years we have witnessed several studies using a paraphernalia of approaches from traditional rational design to in silico techniques - as means of gaining an insight into critical drug targets, or discovering new drug candidates in both known and unexplored chemical space. Screening techniques are very popular in that they are capable of delivering early lead compounds. These serve as starting points for novel opportunities that would not be considered under the precepts of de novo drug design. Virtual screening in particular has surely been playing its part in this quest, but is not faring as well in the field as could have been hoped. However, there is much room for improvement in this field and it should not be written off just yet. Within the past year, three major breakthrough studies in the area of malaria have been made publically available screenings from GlaxoSmithKline, St Jude Children's Research Hospital and Novartis [3-5]. The high-throughput screening (HTS) of GSK's two million-compound library has brought to light approximately 13,500 compounds with the goal of fostering collaborations in drug discovery among different groups and ultimately deliver new medicines to fight malaria [4]. The preference for chemotypes with novel mechanisms of action is widely accepted [6] and with GSK's database available, the search for an efficacious antimalarial will be boosted. This paradigm shift certainly involves risks for the company, but should open up new roads in drug discovery against malaria, and may be fruitful in the long run.

However, is this a one-off situation, or is it going to be followed by other big pharmaceutical companies? And will anyone apply this approach to other severe diseases where the impact on revenue losses is minimal and the emergency need for a cure justifies this action? The current signs are that this open-innovation strategy is to be followed, for neglected diseases, by other major companies. However, it will require a great deal of effort, good will and a new patent system in order to be implemented successfully in the future.

As far as new chemotypes go, the spiroindolone NITD609 from Novartis [3] is the perfect example. It has a single-digit nanomolar $\mathrm{IC}_{50}$ value and a distinct mechanism of action from that of marketed antimalarials; it inhibits protein synthesis by blocking the P-type cationtransporter ATPase4, and complies with all criteria for an antimalarial drug [3]. In addition, the HTS of Guiguemde et al. provided the global

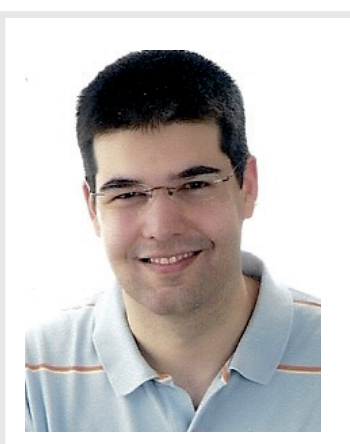

Tiago Rodrigues

Author for correspondence: Faculty of Pharmacy, University of Lisbon, Av. Prof. Gama Pinto 1649-003 Lisbon, Portugal Tel.: +35। 217946400 (ext. 14357) E-mail: trodrigues@ff.ul.pt

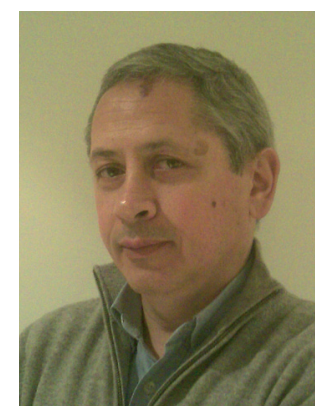

Rui Moreira

Faculty of Pharmacy, University of Lisbon, Portugal

Authors continuted overleaf... 


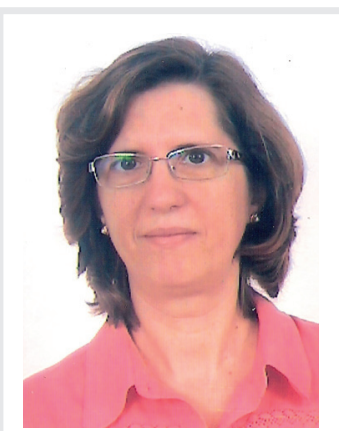

Francisca Lopes

Faculty of Pharmacy, University of Lisbon, Portugal scientific community with several other chemotypes displaying activity against $P$. falciparum dihydroorotate dehydrogenase (PfDHODH), hemozoin formation and $P$. falciparum falcipain-2 [5]. Despite the latter two being relatively known and studied, $P f \mathrm{DHODH}$ represents a novel druggable target for which outstanding results have been achieved [7-9].

What is also striking is the increasing importance and attention given to drug targets directly or indirectly related to de novo pyrimidine biosynthesis. A close look into some of the projects in the lead-generation phase being funded by MMV reveals that approximately half are related to this pathway and one (MK4815) is in the preclinical stage [102]. The parasite cannot salvage preformed pyrimidine bases or nucleosides from its host and, thus, is entirely dependent on de novo biosynthesis $[10,11]$. Pf DHODH is the fourth enzyme in this pathway and its blockage shuts off the downstream production of biomolecules necessary for parasitic multiplication, leading to their death. In an indirect manner, blockage of the mitochondrial electron transport chain (mtETC) results in the same outcome. In this case, the goal is achieved by inhibiting the maintenance of a constant pool of ubiquinone, which is used as cofactor by $P f \mathrm{DHODH}$. GSK has a pyridone pipeline whose target is the $b c_{1}$ complex from the mtETC. It is also remarkable that a large number of hits from the new 13,500 compound library is predicted to act through the mtETC and it will be interesting to see whether new inhibitors of this metabolic pathway are discovered in the near future.

The liver stage of malarial parasites' life cycle also appears to be an interesting target, as it precedes the blood stage, where clinical symptoms arise [12]. Furthermore, there is a relatively small parasite load in the liver compared with that of the blood, which theoretically facilitates the suppression of the infection before it reaches the blood [13]. Relapses of malaria may occur, and are caused by the reactivation of dormant hypnozoites, which initiate a new pre-erythrocytic stage, as in the case of a Plasmodium vivax infection. This is also a major issue, because this species causes high morbidity in Asia, and hampers the elimination efforts. Currently, a limited number of drugs are available to tackle hypnozoites of Plasmodium. Primaquine was specifically developed to inhibit this stage, but its use has been held back by the risk of acute hemolysis in glucose6-phosphate dehydrogenase-deficient patients. On the other hand, atovaquone is not effective against $P$. vivax and Plasmodium ovale dormant stages, and is responsible for a swift selection of mutants in blood stages of $P$. falciparum [14]. What is appealing, is the fact that these two drugs are known to act via interaction with the mitochondrial function, as well as other molecules that have shown usefulness against the liver stage. It makes sense that compounds interfering with the biosynthesis of pyrimidines play a major role in inhibiting the development of merozoites and this pathway has actually been shown to be upregulated at this stage of the life cycle [15]. Despite the technical difficulties in testing compounds against the liver stages of the infection, it would be interesting to see whether the newly available databases will afford compounds with the added value of displaying activity against both blood and liver stages. That would certainly propel antimalarial research to another level, particularly if HTS is applied.

\section{Financial \& competing interest disclosure}

The authors have no relevant affiliations or financial involvement with any organization or entity with a financial interest in or financial conflict with the subject matter or materials discussed in the manuscript. This includes employment, consultancies, honoraria, stock ownership or options, expert testimony, grants or patents received or pending, or royalties.

No writing assistance was utilized in the production of this manuscript.

\section{Bibliography}

1 Carrara VI, Zwang J, Ashley EA et al. Changes in the treatment responses to artesunatemefloquine on the northwestern border of Thailand during 13 years of continuous deployment. PloS One 4(2), e4551 (2009).

2 Ekland EH, Fidock DA. In vitro evaluations of antimalarial drugs and their relevance to clinical outcomes. Int. J. Parasitol. 38(7), 743-747 (2008).
3 Rottmann M, McNamara C, Yeung BKS et al. Spiroindolones, a potent compound class for the treatment of malaria. Science 329(3), 1175-1180 (2010).

4 Gamo FJ, Sanz LM, Vidal J et al. Thousands of chemical starting points for antimalarial lead identification. Nature 465(7296), 305-310 (2010).

5 Guiguemde WA, Shelat AA, Bouck D et al. Chemical genetics of Plasmodium falciparum. Nature 465(7296), 311-315 (2010).
6 Wells TNC, Alonso PL, Gutteridge W. New medicines to improve control and contribute to the eradication of malaria. Nat. Rev. Drug Discov. 8(11), 879-891 (2009).

7 Gujjar R, Marwaha A, Mazouni FE et al. Identification of a metabolically stable triazolopyrimidine-based dihydroorotate dehydrogenase inhibitor with antimalarial activity in mice. J. Med. Chem. 52(7), 1864-1872 (2009). 
8 Phillips MA, Gujjar R, Malmquist NA et al. Triazolopyrimidine-based dihydroorotate dehydrogenase inhibitors with potent and selective activity against the malaria parasite Plasmodium falciparum. J. Med. Chem. 51(12), 3649-3653 (2008).

9 Baldwin J, Michnoff CH, Malmquist NA et al. High-throughput screening for potent and selective inhibitors of Plasmodium falciparum dihydroorotate dehydrogenase. J. Biol. Chem. 280(23), 21847-21853 (2005).

10 Gardner MJ, Hall N, Fung E et al. Genome sequence of the human malaria parasite Plasmodium falciparum. Nature 419(6906), 498-511 (2002).

11 Rodrigues T, Lopes F, Moreira R. Inhibitors of the mitochondrial electron transport chain and de novo pyrimidine biosynthesis as antimalarials: the present status. Curr. Med. Chem. 17(10), 929-956 (2010).
12 Prudêncio M, Rodriguez A, Mota MM. The silent path to thousands of merozoites: the Plasmodium liver stage. Nat. Rev. Microbiol. 4(11), 849-856 (2006).

13 Cunha-Rodrigues M, Prudêncio M, Mota MM, Haas W. Antimalarial drugs-host targets (re)visited. Biotechnol. J. 1(3), 321-332 (2006).

14 Mahmoudi N, Garcia-Domenech R, Galvez J et al. New active drugs against liver stages of plasmodium predicted by molecular topology. Antimicrob. Agents Chemother. 52(4), 1215-1220 (2008).
15 Albuquerque SS, Carret C, Grosso AR et al. Host cell transcriptional profiling during malaria liver stage infection reveals a coordinated and sequential set of biological events. BMC Genomics 10, 270 (2009).

\section{Websites}

101 WHO, World Malaria Report (2009). http://whqlibdoc.who.int/ publications/2009/9789241563901_eng.pdf

102 Medicines for Malaria Venture (2010). www.mmv.org 